\title{
Morbid Obesity is Associated to Altered Fatty Acid Profile of Erythrocyte Membranes
}

Gianmattia del Genio $^{1 *}$, Carla Ferreri², Raffaele Marfella1, Dimitri Pournaras ${ }^{3}$, Carel W le Roux ${ }^{2,4}$, Federica del Genio ${ }^{5}$, Limongelli Paolo1, Salvatore Tolone', Ludovico Docimo' and Annibale Alessandro Puca ${ }^{6}$

${ }^{1}$ Department of Geriatrics and Metabolic Diseases, Second University of Naples, Italy

${ }^{2}$ ISOF, Consiglio Nazionale delle Ricerche, Bologna, Italy

${ }^{3}$ Investigative Science, Imperial College London, UK

${ }^{4}$ Diabetes Complications Research Centre, UCD Conway Institute, University College Dublin, Ireland

${ }^{5}$ Center of Esophago-gastric and Bariatric Surgery (E.G.O.), Clinica C.G. Ruesch, Napoli, Italy

6IRCCS Multimedica, Milan, Italy; Dipartimento di Medicina e Chirurgia, Università degli Studi di Salerno, Italy

\begin{abstract}
Background: Morbid obesity is a pathologic condition associated to an increased incidence of several chronic conditions associated to increased risk of mortality. Erythrocyte fatty acid composition has been candidate as a biomarker of oxidative stress and consequently on the rate of aging and ultimately lifespan. To our knowledge, no study has investigated the total spectrum of lipid composition of erythrocyte membranes from obese individuals. The aim of this study was to compare the fatty acid composition of erythrocyte membranes derived from morbidly obese patients with healthy matched controls and long-living descendants.
\end{abstract}

Methods: Gas chromatography was employed to determine fatty acid percentage of erythrocyte membranes from investigated groups.

Results: Erythrocyte membranes from obese subjects had significantly lower percentage of monounsaturated fatty acids $(16.0 \pm 0.8$ vs. $23.0 \pm 4.5, p<0.01)$ and total trans fatty acid $(0.2 \pm 0.1$ vs. $1.3 \pm 1.2, p<0.001)$ than controls Obese fatty acid profiles showed increased percent of total polyunsaturated fatty acid $(36.5 \pm 1.4 \mathrm{vs}$. $27.3 \pm 10.9$, $p<0.001)$, polyunsaturated fatty acid $n-3(5.2 \pm 0.9$ vs. $2.6 \pm 1.8, p<0.001)$ and $n-6(31.3 \pm 1.7$ vs. $25.0 \pm 10.1$, $\mathrm{p}<0.001)$. Interestingly, when obese fatty acid profiles are compared with long-living descendants, the former show opposite trends in the percentages of the main saturated and unsaturated residues of membrane phospholipids compared with nonagenarian offspring.

Conclusion: This study shows that the erythrocyte membranes fatty acid composition of obese subjects differs from general population in a way opposite to the long-living descendants. If confirmed by further investigations, the fatty acid equilibration may become an additional target in the multidisciplinary strategy aimed to treat the obesity epidemic.

Keywords: Morbid obesity; Fatty acid profile; Long-living descendants; Lipidomics; Gas-liquid chromatography

Abbreviations: BMI: Body Mass Index; MUFA: Monounsaturated Fatty Acids; PUFA: Polyunsaturated Fatty Acids; SFA: Saturated Fatty Acids; TFA: Trans Fatty Acids

\section{Introduction}

Life-insurance data and epidemiological studies confirm that increasing degrees of overweight and obesity are important predictors of decreased longevity [1]. Morbid obesity is a pathologic condition associated to an increased incidence of several chronic conditions, including noninsulin-dependent diabetes mellitus, hypertension, cardiovascular disease, obstructive sleep apnea, and all-cause mortality [2]. There is a linear relationship between BMI above 30 and the incidence of these pathologic conditions [3].

Erythrocyte fatty acid composition has been validated as a reliable biomarker of oxidative stress and consequently on the rate of aging and ultimately lifespan $[4,5]$. We have reported an association between a specific fatty acid profile of blood constituents and longevity [6]. In detail, we have found that a combination of high palmitoleic acid (C16:1), low polyunsaturated fatty acids (PUFA) (especially omega 6) and high endogenous trans fatty acids (TFA), that reflect a radical stress activity, are associated with longevity [6]. Elizondo et al. demonstrated a correlation between liver and erythrocyte phospholipids PUFA from patients with obese non-alcoholic fatty liver disease and proposed the fatty acid in erythrocytes membranes as reliable biomarker of derangements in liver lipid metabolism in obese patients [7]. Petrescu et al. comparing the uptake kinetics of $9,10-[3 \mathrm{H}]$ oleic acid by omental adipocytes isolated from obese and non-obese patients undergoing laparoscopic procedures, observed that in the obese subjects long-chain fatty acid (LCFA) uptake was up-regulated, suggesting that alterations in LCFA partitioning may contribute to the obese phenotype [8].

However, to our knowledge no study has investigated the total spectrum of the lipid composition of erythrocyte membranes from obese individuals in an attempt to establish a relationship between obesity and the associated pathologic conditions. Primary endpoint of this study was to compare the lipid composition in terms of the

${ }^{*}$ Corresponding author: Prof. Gianmattia del Genio, Division of Bariatric Surgery, Università degli studi di Napoli SUN, Via Pansini, 5 I-80131 Napoli, Italy, Tel: +393397008292; E-mail: gdg@doctor.com

Received June 30, 2015; Accepted July 16, 2015; Published July 20, 2015

Citation: del Genio G, Ferreri C, Marfella R, Pournaras D, le Roux CW, et al (2015) Morbid Obesity is Associated to Altered Fatty Acid Profile of Erythrocyte Membranes. J Diabetes Metab 6: 582. doi:10.4172/2155-6156.1000582

Copyright: (c) 2015 del Genio G, et al. This is an open-access article distributed under the terms of the Creative Commons Attribution License, which permits unrestricted use, distribution, and reproduction in any medium, provided the original author and source are credited. 
percentage content of various fatty acids of erythrocyte membranes derived from morbid obese patients selected to bariatric surgery versus healthy matched controls.

\section{Materials and Methods}

\section{Subject characteristics}

Experimental subjects: A total of 21 morbid obese adult patients (12 women; median age, 46 years [23-64]) were enrolled for the fatty acid profile study after that the institutional review board approved the clinical protocol and all subjects involved signed an informed consent form. This study was conducted in accordance with the ethical principles that have their origins in the Declaration of Helsinki and approved by the local institutional review board. Before subjects entered the study, specific informed consent was obtained from each.

The subjects were pooled from a group of consecutive patients selected for a bariatric procedure at the Interuniversity Center for Obesity and Eating Disorder (CISRO), according to the interdisciplinary European guidelines for surgery for morbid obesity [9].

At entry, all patients underwent a clinical evaluation to determine the presence and severity of associated medical conditions according to pre-specified primary and secondary outcome measures. Dietary history, food habits, and previous weight loss efforts were accurately evaluated by means of an interview. All participants completed a standardized dietary support program for 4 months before being enrolled in the study, as described elsewhere [10]. In brief, at the first visit, patients were instructed by the dietician on how to fill in their food and activity diaries. After a week, they were given a low calorie diet, which was tailored to each patient's resting energy expenditure, food and activity diary, and metabolic data. The patients were instructed to gradually increase their physical activity by walking outdoors up to $30 \mathrm{~min}$ a day. Each patient was seen on a biweekly basis. Initial assessment included anthropometric measurements, hematobiochemical examination, homeostasis model assessment, analysis of body composition, as previously described [10].

\section{Anthropometric measurements}

Participants fasted overnight and then were weighed barefoot and in light clothing to the nearest $0.1 \mathrm{~kg}$. Height was measured using a fixed wall stadiometer; height and weight were recorded and BMI (kg/ $\mathrm{m}^{2}$ ) was calculated. Body composition was assessed by bioimpedance analysis (BIA) (Tanita TBF-300MA Body Composition Analyzer, Tanita Corporation, Tokyo, Japan). Measurements were performed at an ambient temperature of $22-24^{\circ} \mathrm{C}$ after voiding and after being in the supine position for $20 \mathrm{~min}$. Height, sex, and age were entered manually; weight was recorded automatically with $0.5 \mathrm{~kg}$ as an adjustment for weight of clothes $[10,11]$. The Tanita software uses in-built prediction equations to estimate fat mass (FM) and fat-free mass (FFM). All patients underwent a comprehensive preoperative evaluation conducted by a behaviourist with expertise in bariatric patient management. This assessment included personal and social history, history of psychiatric problems, current living situation, and support system. None of the participants had any evidence of psychiatric diseases. Patients were considered eligible after evaluation of esophagogastroduodenoscopy, spirometry, and abdominal ultrasound. Patients with type 2 diabetes and hypertension were excluded [10].

\section{Control subjects}

A total of 30 healthy control subjects ( 15 women, median age 48 [22-62]) were enrolled for the fatty acids profile. The control group included healthy normal weight subjects (mean BMI $21.5 \pm 3[18,5$ $25]$ ) in absence of any known medical condition or pathology. There is no reason to believe that there was any sort of bias in favour of a specific diet or physical activity during the recruitment of the group [6].

\section{Lipid isolation from erythrocyte membranes and GC analysis}

Venous blood samples were collected into EDTA vacutainers and centrifuged immediately after collection at $1000 \mathrm{~g}$ for $10 \mathrm{~min}$ at $4^{\circ} \mathrm{C}$. Supernatant plasma was then removed. Separated erythrocytes were suspended in pure water and the mixture vortexed and subsequently centrifuged at $14,000 \mathrm{~g}$ for $30 \mathrm{~min}$ at $4^{\circ} \mathrm{C}$ to separate membrane pellets from the mixture.

Bligh and Dyer [12] performed phospholipids extraction from pellets according to method. The total phospholipid fraction was treated with $\mathrm{KOH} / \mathrm{MeOH}$ solution $(0.5 \mathrm{M})$ for $10 \mathrm{~min}$ at room temperature, and fatty acid methyl esters (FAME) were subsequently extracted with $n$-hexane. GC analysis of released fatty acids showed separation of all fatty acids and their isomers. This was confirmed by comparison with commercially available references and with a library of geometrical trans monounsaturated fatty acids (MUFA) and PUFA obtained by thiyl radical-catalyzed reaction of naturally occurring lipids. This technique has been used by others [13].

\section{Analysis of fatty acids of erythrocyte membranes}

Analysis of the erythrocyte membranes' fatty acid components was performed by evaluating the fatty acid percentages and correlating the percentages of the main saturated and unsaturated residues of membrane phospholipids, together with the sums of saturated fatty acids (SFA), MUFA, and PUFA n-3 and n-6 residues and some indicative ratios (SFA/MUFA, C16:1/C16:0; C18:1/C18:0). In addition, the conditions for analytical detection allowed some trans-fatty acid isomers to be evaluated, which are relative to the isomerization of fatty acids in cell membranes due to an endogenous free radical process.

Moreover, two indices were calculated corresponding to the peroxidation index (PI), which results from the following equation [14]: PI $=[(\%$ monoenoic $\times 0.025)+(\%$ dienoic $\times 1)+(\%$ trienoic $\times$ $2)+(\%$ tetraenoic x 4) + (\%pentaenoic x 6) + (\%hexaenoic x 8)], and the unsaturation index (UI), also known as the index of hydrogen deficiency, which is given by the equation: $\mathrm{UI}=[(\%$ Monoenoic $\mathrm{x} 1)+$ $(\%$ Dienoic $\times 2)+(\%$ Trienoic $\times 3)+(\%$ Tetraenoic $\times 4)+(\%$ Pentaenoic $x 5)+(\%$ Hexaenoic $x 6)]$. These indices allow the study of the influence of the double bond content in the cell membranes of morbid obese subjects and in controls, together with the susceptibility to oxidative degradation.

\section{Statistical analysis}

Statistical analysis was performed using SPSS for Windows (version 17; SPSS Inc., Chicago, IL. USA). Descriptive data are presented as mean values \pm standard deviation (SD), median and interquartile [25$75^{\text {th }}$ range, unless otherwise indicated. An unpaired t-test was generally used for group comparisons. When normality and equal variance analyses failed, a Mann-Whitney ranks sum test was used instead of an unpaired t-test. For all tests, a two-sided $\mathrm{P}<0.05$ was considered statistically significant.

\section{Results}

The cohort patients in the present study exhibited a significant difference of BMI, FM and FFM when compared to control group, as reported in Table 1. 


\begin{tabular}{|c|c|c|c|}
\hline & $\begin{array}{c}\text { Morbid Obese } \\
(\mathbf{n = 2 1 )}\end{array}$ & $\begin{array}{c}\text { Healthy } \\
(\mathbf{n = 3 0 )}\end{array}$ & $\mathbf{P}^{*}$ \\
\hline Weight $[\mathrm{Kg}]$ & $146.3 \pm 29.1$ & $65.0 \pm 13.9$ & $<.001$ \\
\hline BMI $\left[\mathrm{Kg} / \mathrm{m}^{2}\right]$ & $51.3 \pm 11.6$ & $21.5 \pm 3.0$ & $<.001$ \\
\hline Excess body weight [\%] & $98 \pm 19.7$ & $8.2 \pm 5.7$ & $<.001$ \\
\hline FM [Kg] & $66.2 \pm 14.5$ & $26.4 \pm 9.2$ & $<.001$ \\
\hline FM [\%] & $50.2 \pm 10.3$ & $40.6 \pm 5.5$ & $<.001$ \\
\hline FFM [Kg] & $65.8 \pm 7.6$ & $38.6 \pm 6.4$ & $<.001$ \\
\hline FFM [\%] & $49.2 \pm 7.3$ & $59.3 \pm 8.1$ & $<.001$ \\
\hline
\end{tabular}

*Values are mean presented as \pm SD. Abbreviations: FM, fat mass; FFM, fat-free mass. " $P$ value cohort vs. control group

Table 1: Clinical characteristics, body size, densitometric variables of morbid obese subjects vs. healthy control subjects.

\begin{tabular}{|c|c|c|c|}
\hline & Obese patients (21) & Matched control (30) & \\
\hline Variable & Mean \pm SD & Mean \pm SD & p - value \\
\hline C16:0 & $31.3 \pm 2.1$ & $29.4 \pm 6.3$ & - \\
\hline C16:1 & $0.5 \pm .20$ & $1.9 \pm 1.8$ & $<.001$ \\
\hline C18:0 & $14.5 \pm 1.4$ & $16.9 \pm 5.3$ & 0.03 \\
\hline C18:1 & $15.5 \pm 0.8$ & $21.3 \pm 3.2$ & $<.001$ \\
\hline SFA & $45.8 \pm 1.4$ & $46.3 \pm 9.3$ & - \\
\hline MUFA & $16.0 \pm 0.8$ & $23.0 \pm 4.5$ & $<.01$ \\
\hline C18:2 & $12.0 \pm 1.1$ & $10.4 \pm 3.0$ & 0.02 \\
\hline $\mathrm{C} 20: 3$ & $2.0 \pm .4$ & $1.4 \pm 1.0$ & 0.008 \\
\hline C20:4 & $17.4 \pm 1.6$ & $12.9 \pm 7.2$ & 0.003 \\
\hline C20:5 & $0.5 \pm .1$ & $0.9 \pm 1.1$ & - \\
\hline C22:6 & $4.7 \pm 0.8$ & $1.7 \pm 1.3$ & $<.001$ \\
\hline n3 PUFA & $5.2 \pm 0.9$ & $2.6 \pm 1.8$ & $<.001$ \\
\hline n6 PUFA & $31.3 \pm 1.7$ & $25.0 \pm 10.1$ & $<.001$ \\
\hline Total PUFA & $36.5 \pm 1.4$ & $27.3 \pm 10.9$ & $<.001$ \\
\hline TC 18:1 & $0.2 \pm 0.1$ & $0.3 \pm 0.3$ & 0.07 \\
\hline TC20:4 & $0.03 \pm 0.03$ & $1.0 \pm 1.2$ & $<.001$ \\
\hline Total TFA & $0.2 \pm 0.1$ & $1.3 \pm 1.2$ & $<.001$ \\
\hline SFA/MUFA & $2.9 \pm 0.2$ & $2.1 \pm 0.6$ & $<.001$ \\
\hline C16:1/16:0 & $0.02 \pm 0.01$ & $0.1 \pm 0.1$ & 0.002 \\
\hline C18:1/18:0 & $1.1 \pm 0.1$ & $1.4 \pm 0.4$ & $<.001$ \\
\hline
\end{tabular}

Table 2: Lipid composition of erythrocyte membranes derived from obese patients and healthy controls.

There were differences in the fatty acid composition of erythrocyte membranes derived from morbid obese versus matched controls (Table 2).

Specifically, erythrocyte membranes from nonagenarian offspring had significantly lower content of MUFA (16.0 \pm 0.8 vs. $23.0 \pm 4.5$, $\mathrm{p}<0.01)$ and higher of PUFA $(36.5 \pm 1.4$ vs. $27.3 \pm 10.9, \mathrm{p}<0.001)$ than controls. In particular, the experimental subjects had a lower content of $\mathrm{C} 16: 1 \mathrm{n}-7(0.5 \pm 0.20$ vs. $1.9 \pm 1.8, \mathrm{p}<0.001)$, trans $\mathrm{C} 18: 1 \mathrm{n}-9(15.5 \pm$ 0.8 vs. $21.3 \pm 3.2, \mathrm{p}<0.001)$, and total trans $\mathrm{FA}(0.2 \pm 0.1$ vs. $1.3 \pm 1.2$, $\mathrm{p}<0.001$ ), than matched controls (Table 1). The total PUFA increase was due to the significant increase of C18:2, C20:3, C20:4, C22:6, n-3 PUFA, and n-6 PUFA, only C20:5 remained unchanged.

It is notably, to see that in the obese group either the PI (126.0 \pm 7.9 vs. $84.5 \pm 39.2, \mathrm{p}<0.001)$ or the UI $(146.0 \pm 6.1$ vs. $114.3 \pm 38.4$, $\mathrm{p}<0.001)$ were found to be significantly higher than that in the control groups (Table 3 ).

\section{Discussion}

A different composition of lipids types (i.e. SFA, MUFA and
PUFA), as constituents of the cellular membrane, influences its fluidity and permeability and consequently the disease susceptibility [15].

Data from our study clearly demonstrated that in the human obese population the ratio of MUFA and PUFA is sturdily changed. This was due to a parallel reduction of MUFA (especially C16:1 and C18:1) and increase of PUFA. This disequilibrium of polyunsaturated fatty acid metabolism seems to contribute to an excessive adipose tissue development and represents itself an emerging risk factor for obesity [16]. This is consistent with the diet-induced reduction of pro-inflammatory fatty acids profile, we recently reported in coeliac patients [17]. Indeed, in obese patients the significantly higher levels of C20:3 ( $\mathrm{p}=0.008)$ and C20:4 n-6 ( $\mathrm{p}=0.003)$, both PUFA, in erythrocyte membranes of morbid obese group induce a greater membrane peroxidizability. In fact, the PI and UI were found significantly increased in obese subjects compared to controls $(p<0.001)$. In addition, there was a significantly lower level of trans C18:1 n-9 in the erythrocyte membranes of obese compared with controls $(\mathrm{p}<0.001)$, which may indicate a lower exposition to radical stress.

The lower content of palmitoleic acid and oleic acid may compensate for the increased permeability created by the lower percentage of TFA and possibly for the major content in PUFA in erythrocyte membranes [18]. Indeed, the inverse relationship between polyene-containing lipid molecular species and monoene counterparts has been recently documented in cultured cells [19].

We have recently demonstrated that erythrocyte membranes derived by an identical process from nonagenarian offspring have a different lipid composition to that of the general population, in a manner indicative of reduced lipid peroxidation, increased membrane integrity and cytoprotective response [6]. If we compare data of this study with those of nonagenarian offspring and controls, we observe that erythrocyte membranes from the obese group showed opposite differences than nonagenarian offspring in fatty acids percentage. In particular, nonagenarian offspring showed a significantly major content of total MUFA (especially C16:1), total SFA, (especially C18:0), and total TFA (especially trans C18:1) and a minor content of total PUFA, (especially the PUFA n-6, C22: 6, C18:2 and C20:4) compared to control and obese subjects (Figure 1). Furthermore, we observed that both PI and UI indexes were considerably lower in nonagenarians offspring than controls group showing again an opposite trend compared to the obese subjects.

These comparisons underline the importance of membrane fatty acids in determining the risk of obesity associated disease. If confirmed by further investigations, the fatty acid equilibration may become an additional target in the multidisciplinary strategy aimed to treat the obesity epidemic.

\section{Conflicts of Interest Disclosure Statement}

The authors declare that they have no conflict of interest, financial ties or funding source to disclose.

\begin{tabular}{|c|r|c|c|}
\hline & Obese patients (21) & Matched control (30) & \multirow{2}{*}{ p - value } \\
\cline { 1 - 3 } Variable & Mean \pm SD & Mean \pm SD & \\
\hline PI & $126.0 \pm 7.9$ & $84.5 \pm 39.2$ & $<.001$ \\
\hline UI & $146.0 \pm 6.1$ & $114.3 \pm 38.4$ & $<.001$ \\
\hline
\end{tabular}

Data are presented as mean \pm S.D. Abbreviations: PI, Peroxidation Index; UI, Unsaturation index (see methods)

Table 3: Comparison of Peroxidation index (PI) and Unsaturation index (UI) of morbid obese subjects and healthy controls. 
Citation: del Genio G, Ferreri C, Marfella R, Pournaras D, le Roux CW, et al. (2015) Morbid Obesity is Associated to Altered Fatty Acid Profile of Erythrocyte Membranes. J Diabetes Metab 6: 582. doi:10.4172/2155-6156.1000582

Page 4 of 4

$\square$ Obese $\square$ Controls $\square$ Nonagenarians

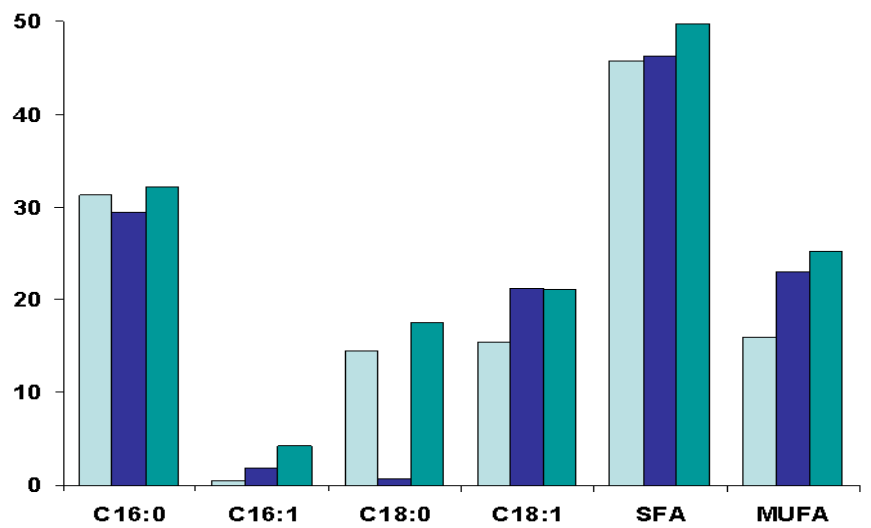

Figure 1: Total MUFA, total SFA, and total TFA, and a minor content of total PUFA compared to control and obese subjects.

\section{Sources of Funding}

Drs. Gianmattia del Genio et al. have no conflict of interest, financial ties or funding source to disclose. Prof. Carel le Roux is supported by Science Foundation Ireland 12/YI/B2480.

\section{References}

1. Lew EA (1985) Mortality and weight: insured lives and the American Cancer Society studies. Ann Intern Med 103: 1024-1029.

2. Kopelman PG (2000) Obesity as a medical problem. Nature 404: 635-643

3. Willett WC, Dietz WH, Colditz GA (1999) Guidelines for healthy weight. N Engl J Med 341: 427-434.

4. Caprari P, Scuteri A, Salvati AM, Bauco C, Cantafora A, et al. (1999) Aging and red blood cell membrane: a study of centenarians. Exp Gerontol 34: 47-57.

5. Rabini RA, Moretti N, Staffolani R, Salvolini E, Nanetti L, et al. (2002) Reduced susceptibility to peroxidation of erythrocyte plasma membranes from centenarians. Exp Gerontol 37: 657-663.

6. Puca AA, Andrew P, Novelli V, Anselmi CV, Somalvico F, et al. (2008) Fatty acid profile of erythrocyte membranes as possible biomarker of longevity. Rejuvenation Res 11: 63-72.
7. Elizondo A, Araya J, Rodrigo R, Poniachik J, Csendes A, et al. (2007) Polyunsaturated fatty acid pattern in liver and erythrocyte phospholipids from obese patients. Obesity (Silver Spring) 15: 24-31.

8. Petrescu O, Fan X, Gentileschi P, Hossain S, Bradbury M, et al. (2005) Longchain fatty acid uptake is upregulated in omental adipocytes from patients undergoing bariatric surgery for obesity. Int J Obes (Lond) 29: 196-203.

9. Fried M, Hainer V, Basdevant A, Buchwald H, Deitel M, et al. (2007) Interdisciplinary European guidelines for surgery for severe (morbid) obesity. Obes Surg 17: 260-270.

10. del Genio F, Alfonsi L, Marra M, Finelli C, del Genio G, et al. (2007) Metabolic and nutritional status changes after $10 \%$ weight loss in severely obese patients treated with laparoscopic surgery vs integrated medical treatment. Obes Surg 17: 1592-1598.

11. Del Genio F, Del Genio G, De Sio I, Marra M, Alfonsi L, et al. (2009) Noninvasive evaluation of abdominal fat and liver changes following progressive weight loss in severely obese patients treated with laparoscopic gastric bypass. Obes Surg 19: 1664-71.

12. Bligh EG, Dyer WJ (1959) A rapid method of total lipid extraction and purification. Can J Biochem. Physiol 37: 911-917.

13. Ferreri C, Kratzsch S, Brede O, Marciniak B, Chatgilialoglu C (2005) Trans lipid formation induced by thiols in human monocytic leukemia cells. Free Radic Bio Med 38: 1180-1187.

14. Witting LA, Horwitt MK (1964) Effect of Degree of Fatty Acid Unsaturation in Tocopherol Deficiency-Induced Creatinuria. J Nutr 82: 19-33.

15. Vance DE, Vance JE (2002) Biochemistry of lipids, lipoproteins and membranes ( $4^{\text {th }}$ edtn) Elsevier Science BV: Amsterdam.

16. Ailhaud G, Guesnet P, Cunnane SC (2008) An emerging risk factor for obesity: does disequilibrium of polyunsaturated fatty acid metabolism contribute to excessive adipose tissue development? Br J Nutr 100: 461-470

17. Riezzo G, Ferreri C, Orlando A, Martulli M, D’Attoma B, et al. (2014) Lipidomic analysis of fatty acids in erythrocytes of coeliac patients before and after a gluten-free diet intervention: a comparison with healthy subjects. Br J Nutr 112: 1787-1796.

18. Ferreri C, Pierotti S, Barbieri A, Zambonin L, Landi L, et al. (2006) Comparison of phosphatidylcholine vesicle properties related to geometrical isomerism. Photochem Photobiol 82: 274-280.

19. Balogh G, Horvath I, Liebisch G, Maslyanko A, Nagy E, et al. (2006) Variations in culture conditions profoundly alter the lipid composition of mammalian cells: Implications for the stress response modulation. (Abstract) Chem Phys Lipids 143: A67. 